\title{
Estudos sobre Aplicações Conjuntas de Herbicidas e Nematicidas Sistêmicos na Eficácia dos Nematicidas em Cana-de-Açúcar
}

\author{
Andrea C. B. Barros ${ }^{1}$, Romero M. Moura ${ }^{1}$ \& Elvira M. R. Pedrosa ${ }^{2}$ \\ ${ }^{1}$ Departamento de Agronomia, ${ }^{2}$ Departamento de Tecnologia Rural, Universidade Federal Rural de Pernambuco, \\ Dois Irmãos, CEP 52171-900, Recife, PE, e-mail: baltarbarros@aol.com
}

(Aceito para publicação em 03/02/2006)

Autor para correspondência: Andréa Cristina Baltar Barros

BARROS, A.C.B., MOURA, R.M. \& PEDROSA, E.M.R. Estudos sobre aplicações conjuntas de herbicidas e nematicidas sistêmicos na eficácia dos nematicidas em cana-de-açúcar. Fitopatologia Brasileira 31:291-296. 2006.

\begin{abstract}
RESUMO
Com o objetivo de avaliar uma possível influência da aplicação conjunta de nematicidas sistêmicos e herbicidas sobre a eficácia dos nematicidas, no controle de Meloidogyne spp. e Pratylenchus zeae em cana-de-açúcar (híbrido de Saccharum spp.) no Nordeste, instalaram-se dois experimentos, um irrigado e outro não irrigado, ambos em canaviais nordestinos, há muito cultivados e comprovadamente infestados por fitonematóides. A variedade utilizada foi SP79-1011, os nematicidas aldicarb e terbufós e os herbicidas diuron, ametrina, oxyfluorfen e pendimetalin, aplicados no plantio, nas doses recomendadas comercialmente. As avaliações fundamentaram-se nos níveis populacionais dos nematóides observados em três diferentes momentos, e nos fatores de reprodução. Avaliaram-se também o desenvolvimento das plantas, a produtividade agrícola e dois parâmetros tecnológicos. Os resultados demonstraram que não houve efeito dos herbicidas na eficácia dos nematicidas, nem foram significativas as diferenças em relação à produtividade, exceto na área irrigada, para perfilhamento nos tratamentos com herbicidas, e número de colmos e perfilhos, nos tratamentos com nematicidas em relação à testemunha. Os nematicidas e herbicidas não afetaram os níveis de Pol e PCC do caldo, não interferindo, portanto, na produtividade industrial. Houve interação estatística entre nematicida e herbicida para P. zeae na rizosfera, na área irrigada, com a menor população do parasito encontrada na combinação aldicarb + pendimetalin.
\end{abstract}

Palavras-chave adicionais: Saccharum sp., nematóides, controle químico, Meloidogyne, Pratylenchus.

\begin{abstract}
Studies on the effect of simultaneous application of herbicide and systemic nematicide on nematicide efficacy in sugarcane fields

The effect of interaction between herbicides (diuron, ametrina, oxyfluorfen, and pendimetalin) and systemic nematicides (aldicarb and terbufos) on growth and yield of sugarcane (hybrid of Saccharum spp.) variety SP79-1011 in soil naturally infested with Meloidogyne incognita, M. javanica and Pratylenchus zeae was evaluated in two different experiments: irrigated and not irrigated, in Northeastern Brazil. Evaluations were based upon nematode population densities and reproduction factor, plant growth, crop productivity and two technological parameters. There was no interaction between herbicides and nematicides in the sense of reducing nematicide efficiency, except in an irrigated experiment for number of stalks in herbicide treatments and number of stems and stalks in nematicide treatments. Nematicides and herbicides did not affect Pol and PCC levels at harvesting. There was interaction between nematicide and herbicide for $P$. zeae in the rhyzosphere in the irrigated experiment, with the lowest population found in aldicarb + pendimetalin interaction.

Additional keywords: Saccharum sp., plant-parasitic nematodes, chemical control, Meloidogyne, Pratylenchus.
\end{abstract}

\section{INTRODUÇÃO}

Ousodenematicidas sistêmicostem sidorecomendado para a cultura da cana-de-açúcar (híbridos de Saccharum spp.) por muitos pesquisadores (Novaretti et al., 1985; Spaull \& Cadet, 1990; Moura, 1995; Garcia et al., 1997). Os estudos nos quais se aplicaram nematicidas no plantio de diversas variedades constataram que esses produtos contribuíram para aumentos significativos de produtividade agrícola da cultura em solos infestados por nematóides (Dinardo-Miranda et al., 1995; Moura, 1995; Barros et al., 2000). Diante desses fatos, somados à existência no mercado de nematicidas sintéticos, possíveis de serem empregados em cana-de-açúcar, tornou-se crescente o uso desses produtos que são aplicados no sulco de plantio. Por outro lado, plantas daninhas também podem causar significativas reduções de produtividade, principalmente pela competição por água e nutrientes. Na maioria das áreas cultivadas com cana-de-açúcar, o controle de plantas daninhas é realizado com aplicação de herbicidas em pré-emergência, logo após a aplicação do nematicida. Entretanto, tem sido observado por agricultores que as aplicações conjuntas de nematicidas e herbicidas podem induzir maior severidade de sintomas de fitotoxidade, fato relatado com frequencia (Blanco et al. 
1980; Copersucar 1982; Fugiwara \& Christoffoleti 1996; Dinardo-Miranda et al. 2001). Não há dados na literatura sobre efeitos de herbicidas na eficácia de nematicidas sistêmicos por interações entre produtos. Por outro lado, tem sido constante a reclamação de agricultores de que, na prática, nem sempre a aplicação de nematicidas em cana-de-açúcar traz resultados satisfatórios. O objetivo da presente pesquisa foi avaliar o possível efeito da aplicação conjunta de dois nematicidas sistêmicos e quatro herbicidas na eficicácia dos nematicidas em canade-açúcar, utilizando-se produtos de uso freqüente pela indústria sucroalcooleira do Nordeste, observando-se também efeitos de fitotoxidade.

\section{MATERIAL E MÉTODOS}

Conduziram-se dois experimentos em áreas de produção comercial de cana-de-açúcar, pertencentes à Destilaria GIASA (Gramami Industrial e Agrícola S.A.), no município de Pedras de Fogo, Paraíba, um irrigado por aspersão (novembro de 2000 a novembro de 2001) e outro não irrigado (agosto de 2001 a agosto de 2002). As áreas experimentais estavam naturalmente infestadas por fitonematóides, compredominânciadasespéciesMeloidogyne incognita (Kofoid \& White) Chitwood Meloidogyne javanica (Treub) Chitwood, e Pratylenchus zeae Graham, sendo a escolha dessas áreas fundamentada em dados populacionais dos fitonematóides no solo, previmente obtidos por uma amostragem do canavial, coletando-se, para tanto, amostras de solo e raízes para extração, identificação e quantificação de gêneros e espécies. Os resultados indicaram estarem as áreas adequadamente infestadas para os experimentos. A variedade utilizada foi a SP79-1011. Os nematicidas estudados, devidamente registrados para emprego na cultura da cana-de-açúcar, foram aplicados no fundo do sulco de plantio e tinham as denominações técnicas aldicarb (carbamato, solubilidade $9000 \mathrm{ppm}$, de nome comercial Temik) e terbufós (organofosforado, solubilidade $5 \mathrm{ppm}$, de nome comercial Counter 150G). Esses produtos foram aplicados nas doses de $10 \mathrm{~kg} / \mathrm{ha}$ e $20 \mathrm{~kg} / \mathrm{ha}$ do produto comercial, respectivamente. Os herbicidas avaliados foram: diuron (Cention SC) 2,5 L/ ha; ametrina (Gesapax 500) 5,0 L/ha; oxyfluorfen (Goal BR) 2,0 L/ha e pendimetalin (Herbadox $500 \mathrm{CE}$ ) 4,0 L/ha, todos aplicados em pré-emergência, logo após o plantio da cana.

O delineamento experimental foi do tipo blocos ao acaso, com 11 tratamentos e cinco repetições, totalizando 55 parcelas para cada experimento. A unidade experimental era um talhão, com sete linhas de $10 \mathrm{~m}$ de comprimento, espaçadas por 1,4 m, sendo as duas externas bordaduras e as três centrais as úteis para coleta de dados. As determinações das populações iniciais $(\mathrm{Pi})$ dos nematóides foram feitas por amostragens de solo, antes da aplicação dos produtos, por ocasião do plantio, no Laboratório de Fitonematologia da Universidade Federal Rural de Pernambuco (UFRPE).
A coleta das amostras foi feita segundo Barker (1985) e o processo de extração pelo método de Jenkins (1964). O período experimental totalizou 12 meses, com as avaliações fundamentadas nos níveis populacionais dos nematóides na rizosfera e nas raízes aos 6 e 12 meses após o plantio e nos fatores de reprodução $(\mathrm{FR}=\mathrm{Pf} / \mathrm{Pi})$ dos nematóides estudados. Após a colheita, dez colmos foram retirados ao acaso, para análise das variáveis relativas à produtividade industrial, representada pelo Pol (\% de sacarose) e PCC (\% de sacarose corrigida), seguindo-se a metodologia padronizada para a indústria açucareira (Fernandez, 2001).

Em relação ao desenvolvimento e produtividade da cana-de-açúcar, foram avaliados perfilhamento aos três meses, número, peso, comprimento e diâmetro dos colmos aos 12 meses após o plantio. Os dois últimos parâmetros foram analisados em 10 colmos retirados ao acaso de cada parcela. As adubações e demais procedimentos fitotécnicos nas duas áreas, irrigada e não irrigada, seguiram a rotina da Empresa. Os dados das estimativas populacionais dos nematóides e da produtividade da cultura foram estatisticamente avaliados pela análise de variância, utilizando-se o Teste de Tukey, para a comparação das médias, no programa estatístico SAS, com os dados das populações dos nematóides transformados em log (x $+1)$.

\section{RESULTADOS E DISCUSSÃO}

Em relação aos indicadores de produtividade, não foram encontrados dados que indicassem incompatibilidade no uso conjunto de nematicida e herbicida. Nos dois experimentos, os resultados dos tratamentos foram estatisticamente equivalentes aos das testemunhas (Tabela 1). Este fato excluiu a possibilidade da ocorrência de efeitos fitotóxicos severos relacionados à aplicação conjunta desses insumos, discordando de observações feitas por outros autores (Blanco et al. 1980; Copersucar 1982; Fugiwara \& Christoffoleti 1996 e Dinardo-Miranda et al., 2001). Ainda em relação à produtividade, as médias das diferentes variáveis foram sempre mais altas na área não irrigada do que na irrigada, que possuía maiores densidades populacionais de $P$. zeae (Tabela 1). As diferenças em solubilidade dos produtos nematicidas, aparentemente, não tiveram influência nos tratamentos. Os herbicidas também não induziram diferenças significativas nos parâmetros de avaliação de produtividade, exceto para número de perfilhos (Tabela 2). Por exemplo, em relação às testemunhas, foram encontradas diferenças que variaram de 20,97 t/ha na aplicação de oxyfluorfen a 35,52 $\mathrm{t} / \mathrm{ha}$ para o produto pendimetalin (Tabela 2). Esses aumentos corresponderam, provavelmente, à maior capacidade de eliminação de ervas daninhas. A análise visual do campo indicou que todos os herbicidas pesquisados foram efetivos no controle das ervas daninhas, não apresentando sintomas de fitotoxidade.

$\mathrm{Na}$ área irrigada, concordando com os dados de Lusvargui et al., 1997 e Moura et al., 1998, a aplicação dos nematicidas sistêmicos isoladamente aumentou o número 
Estudos sobre aplicações conjuntas de herbicidas e nematicidas...

TABELA 1 - Efeito da aplicação conjunta de nematicidas sistêmicos e herbicidas na produtividade da variedade de cana-de-açúcar (Saccharum spp.) SP79-1011, em solos naturalmente infestados por fitonematóides, em área irrigada e não irrigada

\begin{tabular}{|c|c|c|c|c|c|}
\hline \multirow[b]{2}{*}{ Nematicida } & \multicolumn{5}{|c|}{ Produtividade (Colmos) } \\
\hline & \multirow[t]{2}{*}{ la Herbicida } & $\begin{array}{c}\text { Número } \\
\text { total }\end{array}$ & $\begin{array}{l}\text { Peso } \\
(t / h a)\end{array}$ & $\begin{array}{c}\text { Diâmetro }^{1} \\
(\mathrm{~mm})\end{array}$ & $\begin{array}{c}\text { Comp. }{ }^{1} \\
\text { (m) }\end{array}$ \\
\hline & & \multicolumn{4}{|c|}{ Área irrigada } \\
\hline \multirow{6}{*}{ terbufós } & diuron & 67.440 & 48,31 & 23,35 & 1,49 \\
\hline & oxyfluor fen & 71.136 & 51,47 & 23,60 & 1,58 \\
\hline & ametrina & 68.832 & 50,67 & 23,66 & 1,51 \\
\hline & pendimetalin & 76.032 & 57,47 & 23,82 & 1,53 \\
\hline & Sem herbicida & 65.952 & 48,53 & 24,31 & 1,57 \\
\hline & diuron & 70.272 & 49,13 & 23,41 & 1,45 \\
\hline \multirow{4}{*}{ aldicarb } & oxyfluor fen & 66.720 & 47,54 & 24,45 & 1,42 \\
\hline & ametrina & 70.656 & 50,42 & 24,00 & 1,58 \\
\hline & pendimetalin & 72.288 & 59,69 & 24,27 & 1,54 \\
\hline & Sem herbicida & 61.680 & 43,32 & 24,62 & 1,43 \\
\hline \multicolumn{2}{|c|}{ Testemunha } & 56.880 & 40,47 & 24,54 & 1,45 \\
\hline \multicolumn{2}{|c|}{ C.V. $(\%)=$} & 11,59 & 18,77 & 4,12 & 8,02 \\
\hline \multicolumn{6}{|c|}{ Área não irrigada } \\
\hline \multirow{5}{*}{ terbufós } & diuron & 86.064 & 106,32 & 25,06 & 2,43 \\
\hline & oxyfluor fen & 85.720 & 102,96 & 24,28 & 2,51 \\
\hline & ametrina & 88.800 & 104,88 & 24,65 & 2,50 \\
\hline & pendimetalin & 84.720 & 100,08 & 24,98 & 2,46 \\
\hline & Sem herbicida & 84.528 & 103,44 & 24,82 & 2,42 \\
\hline \multirow{5}{*}{ aldicarb } & diuron & 84.672 & 102,24 & 23,75 & 2,40 \\
\hline & oxyfluor fen & 84.816 & 102,96 & 24,72 & 2,42 \\
\hline & ametrina & 87.024 & 97,68 & 24,91 & 2,28 \\
\hline & pendimetalin & 85.872 & 101,76 & 25,49 & 2,40 \\
\hline & Sem herbicida & 85.152 & 99,36 & 24,35 & 2,31 \\
\hline \multicolumn{2}{|c|}{ Testemunha } & 82.104 & 99,12 & 24,88 & 2,35 \\
\hline \multicolumn{2}{|c|}{ C.V. $(\%)=$} & 6,34 & 10,16 & 5,01 & 4,62 \\
\hline
\end{tabular}

${ }^{1}$ Números médios de dez colmos tomados ao acaso. Os dados são médias de cinco repetições.

Não houve diferença significativa entre os tratamentos.

TABELA 2 - Produtividade da variedade de cana-de-açúcar (Saccharum spp.) SP79-1011 na presença ou ausência de quatro herbicidas, independentemente dos tratamentos com nematicida, em solos naturalmente infestados por fitonematóides, em área irrigada

\begin{tabular}{|c|c|c|c|c|c|}
\hline \multirow[b]{2}{*}{ Tratamento } & \multirow[b]{2}{*}{$\begin{array}{c}\mathbf{N}^{0} \text { Perfilhos } \\
\text { (ha) }\end{array}$} & \multicolumn{4}{|c|}{ Produtividade (Colmos/ha) } \\
\hline & & $\begin{array}{c}\text { Número } \\
\text { total }\end{array}$ & $\begin{array}{l}\text { Peso } \\
\text { (T) }\end{array}$ & $\begin{array}{c}\text { Diâmetro }^{1} \\
(\mathrm{~mm})\end{array}$ & $\begin{array}{c}\text { Comp } 1 \\
(\mathrm{~m})\end{array}$ \\
\hline & & $68.856,0 \mathrm{a}$ & $48,72 \mathrm{a}$ & & $1,47 \mathrm{a}$ \\
\hline & & 68.928 & 49,5 & & $1,50 \mathrm{a}$ \\
\hline & & $69.744,0 \mathrm{a}$ & 50 & 2. & $1,55 \mathrm{a}$ \\
\hline & 103. & 74.1 & $58,58 \mathrm{a}$ & 24, & $1,53 \mathrm{a}$ \\
\hline Sem herbicida & $67.608 \mathrm{~b}$ & $61.504,0 \mathrm{a}$ & $44,11 \mathrm{a}$ & $24,49 a$ & $1,48 \mathrm{a}$ \\
\hline C.V. $(\%)=$ & 20,45 & 11,58 & 18,76 & 4,11 & 8,02 \\
\hline
\end{tabular}

${ }^{1}$ Números médios de dez colmos tomados ao acaso. Os dados são médias de cinco repetições. Para cada coluna, médias seguidas da mesma letra não diferem estatisticamente ao nível de $5 \%$ de probabilidade pelo teste de Tukey.

de perfilhos e, conseqüentemente, o de colmos por hectare. Fato semelhante ocorreu na área não irrigada, porém as diferenças não foram significativas. Dados referentes à brotação não foram obtidos na área não irrigada. Ao modo do que ocorre com alta freqüência no Nordeste, essas diferenças
TABELA 3 -. Desempenho da variedade de cana-de-açúcar (Saccharum spp.) SP79-1011 na presença ou ausência de nematicidas sistêmicos, independentemente dos tratamentos com herbicida, em solos naturalmente infestados por fitonematóides, em área irrigada e não irrigada

\begin{tabular}{|c|c|c|c|c|c|}
\hline \multirow[b]{2}{*}{ Tratamento } & \multirow{2}{*}{$\begin{array}{c}\mathbf{N}^{0} \text { Perfilhos } \\
\text { (ha) }\end{array}$} & \multicolumn{4}{|c|}{ Produtividade (Colmos) } \\
\hline & & $\begin{array}{c}\text { Número } \\
\text { total }\end{array}$ & $\begin{array}{c}\text { Peso } \\
\text { (t/ha) }\end{array}$ & $\begin{array}{c}\text { Diâmetro }{ }^{1} \\
(\mathrm{~mm})\end{array}$ & $\begin{array}{c}\text { Comp. } \\
\text { (m) }\end{array}$ \\
\hline \multicolumn{6}{|c|}{ Área irrigada } \\
\hline terbufós & $86.179,2 \mathrm{a}$ & $69.878,4 \mathrm{a}$ & $51,29 \mathrm{a}$ & $23,75 \mathrm{a}$ & $1,53 \mathrm{a}$ \\
\hline aldicarb & $94.156,8 \mathrm{a}$ & $68.323,2 \mathrm{a}$ & $50,00 \mathrm{a}$ & $24,15 \mathrm{a}$ & $1,45 \mathrm{a}$ \\
\hline Testemunha & $61.104,0 \mathrm{~b}$ & $56.880,0 \mathrm{~b}$ & $48,53 \mathrm{a}$ & $24,54 \mathrm{a}$ & $1,48 \mathrm{a}$ \\
\hline C.V. $(\%)=$ & 20,45 & 11,58 & 18,76 & 4,11 & 8,02 \\
\hline \multicolumn{6}{|c|}{ Área não irrigada } \\
\hline terbufós & - & $85.968 \mathrm{a}$ & $103,54 \mathrm{a}$ & $24,76 \mathrm{a}$ & $2,46 \mathrm{a}$ \\
\hline aldicarb & - & $85.507 \mathrm{a}$ & $100,8 \mathrm{a}$ & $24,64 \mathrm{a}$ & $2,36 \mathrm{~b}$ \\
\hline Testemunha & - & $82.104 \mathrm{a}$ & $99,12 \mathrm{a}$ & $24,88 \mathrm{a}$ & $2,35 \mathrm{ab}$ \\
\hline C.V. $(\%)=$ & - & 6,34 & 10,16 & 5,01 & 4,62 \\
\hline
\end{tabular}

${ }^{1}$ Números médios de dez colmos tomados ao acaso. Os dados são médias de cinco repetições. Para cada coluna, médias seguidas da mesma letra não diferem estatisticamente ao nível de $5 \%$ de probabilidade pelo teste de Tukey.

*Dados de número de perfilhos não contabilizados.

constatadas nos dois experimentos não se revelaram quando da aferição dos parâmetros de produtividade, 12 meses após o plantio, caracterizando-se um caso típico de aplicação de nematicida sem sucesso econômico (Tabela 3). O aumento em produtividade induzido pelos nematicidas sistêmicos, quando obtidos, têm sido primordialmente conseqüência do maior número de colmos por hectare, fato constatado nas duas áreas experimentais, sem diferença significativa na área não irrigada. $\mathrm{O}$ aparente efeito tônico e nematicida dos nematicidas sistêmicos, aumentando brotação e perfilhamento, associado ao controle populacional dos fitonematóides nos primeiros meses após o plantio, foi constatado por diversos autores (Dinardo-Miranda et al., 1995; Moura, 1995; Barros et al., 2000).

No que concerne o efeito da aplicação conjunta de herbicida com nematicida sistêmico no momento do plantio, os resultados demonstraram que os herbicidas estudados não

TABELA 4 - Influência de quatro herbicidas na eficiência de dois nematicidas sistêmicos sobre a população média de Pratylenchus zeae presente na rizosfera (nematóide / $300 \mathrm{cc}$ de solo) da variedade de cana-de-açúcar (Saccharum spp.) SP79-1011, na área irrigada

\begin{tabular}{lccc}
\hline \hline \multirow{2}{*}{ Herbicida } & \multicolumn{3}{c}{ Nematicida } \\
\cline { 2 - 4 } & terbufós & aldicarb & $\begin{array}{c}\text { Sem } \\
\text { nematicida }\end{array}$ \\
\hline diuron & $605 \mathrm{aA}$ & $749 \mathrm{abA}$ & - \\
oxyfluor fen & $509 \mathrm{aA}$ & $570 \mathrm{abA}$ & - \\
ametrina & $500 \mathrm{aA}$ & $808 \mathrm{aA}$ & - \\
pendimetalin & $850 \mathrm{aA}$ & $164 \mathrm{bB}$ & - \\
Sem herbicida & $692 \mathrm{aA}$ & $953 \mathrm{aA}$ & $1.422 \mathrm{~A}$ \\
\hline C.V.(\%)=18,42 & & &
\end{tabular}

Os dados são médias de cinco repetições. Para cada coluna e para cada linha, médias seguidas da mesma letra minúscula e maiúscula, respectivamente, não diferem estatisticamente ao nível de $5 \%$ de probabilidade pelo teste de Tukey. Dados apresentados em antilog $\mathrm{x}-1$. 
afetaram a eficácia dos princípios ativos nematicida ao longo do experimento, exceto a interação aldicarb + pendimetalin que demonstrou menor população de $P$. zeae na área irrigada
(Tabela 4). Pelo fato dos resultados terem sido semelhantes nas duas áreas estudadas, foram apresentados apenas os dados referentes à área irrigada. Com essa informação,

TABELA 5 - Número de Pratylenchus zeae na rizosfera e raízes da variedade de canade-açúcar (Saccharum spp.) em área irrigada e não irrigada, na presença ou ausência de dois nematicidas sistêmicos e quatro herbicidas

\begin{tabular}{|c|c|c|c|c|c|c|c|c|}
\hline \multirow{2}{*}{ Nematicida } & \multirow{2}{*}{ Herbicida } & \multicolumn{4}{|c|}{ Rizosfera (300ce de solo) } & \multicolumn{3}{|c|}{ Raízes (50 g ) } \\
\hline & & $\mathbf{P i}$ & P6 & P12 & FR* & P6 & P12 & $\mathbf{F R}^{* *}$ \\
\hline \multicolumn{9}{|c|}{ Área irrigada } \\
\hline \multirow{6}{*}{ terbufós } & diuron & 170 & 1.151 & 1.125 & 7,41 & 4.236 & 7.106 & 1,74 \\
\hline & oxyfluor fen & 140 & 1.553 & 603 & 14,68 & 4.254 & 7.770 & 3,67 \\
\hline & ametrina & 270 & 1.010 & 457 & 11,89 & 4.047 & 8.397 & 2,12 \\
\hline & pendimetalin & 310 & 1.635 & 1.208 & 7,95 & 4.993 & 4.237 & 1,16 \\
\hline & Sem herbicida & 296 & 1.305 & 854 & 5,31 & 3.474 & 6.193 & 1,97 \\
\hline & diuron & 189 & 2.016 & 1.099 & 14,53 & 9.425 & 8.280 & 0,75 \\
\hline \multirow{4}{*}{ aldicarb } & oxyfluor fen & 209 & 2.755 & 320 & 13,77 & 7.447 & 10.080 & 0,99 \\
\hline & ametrina & 238 & 2.016 & 1.099 & 15,21 & 9.426 & 8.280 & 1,32 \\
\hline & pendimetalin & 85 & 455 & 112 & 7,66 & 10.753 & 9.805 & 1,18 \\
\hline & Sem herbicida & 148 & 2.949 & 1.967 & 11,55 & 13.619 & 7.067 & 0,61 \\
\hline Testemunha & & 461 & 4.018 & 1.552 & 8,53 & 14.298 & 8.526 & 0,63 \\
\hline \multicolumn{2}{|l|}{ C.V. $(\%)=$} & \multicolumn{4}{|c|}{18,42} & \multicolumn{2}{|c|}{6,21} & \\
\hline \multicolumn{9}{|c|}{ Área não irrigada } \\
\hline \multirow{5}{*}{ terbufós } & diuron & 17 & 49 & 100 & 7,73 & 1.674 & 1.687 & 1,33 \\
\hline & oxyfluor fen & 38 & 43 & 84 & 6,49 & 801 & 1.707 & 4,53 \\
\hline & ametrina & 36 & 26 & 183 & 8,84 & 1.583 & 1.757 & 1,26 \\
\hline & pendimetalin & 26 & 58 & 45 & 16,1 & 1.310 & 2.438 & 3,51 \\
\hline & Sem herbicida & 33 & 58 & 96 & 7,59 & 1.599 & 2.735 & 2,25 \\
\hline \multirow{5}{*}{ aldicarb } & diuron & 50 & 32 & 75 & 10,82 & 2.748 & 3.423 & 3,24 \\
\hline & oxyfluor fen & 19 & 52 & 42 & 7,47 & 2.559 & 2.532 & 1,05 \\
\hline & ametrina & 37 & 103 & 45 & 5,00 & 2.522 & 2.336 & 1,25 \\
\hline & pendimetalin & 37 & 46 & 27 & 4,34 & 3.875 & 2.268 & 0,66 \\
\hline & Sem herbicida & 22 & 47 & 41 & 8,04 & 2.799 & 3.956 & 1,77 \\
\hline Testemunha & & 29 & 81 & 1383, & & 6.306 & 3.5690 & \\
\hline C.V. $(\%)=$ & & \multicolumn{2}{|c|}{14,54} & & & 8,74 & & \\
\hline
\end{tabular}

Dados apresentados em antilog x - 1. Pi = População inicial; P6 e P12 = População aos seis e 12 meses após o plantio, respectivamente. Não houve diferença significativa entre os tratamentos. *FR $=\mathrm{P} 12 / \mathrm{Pi}$ - Média resultante das médias dos FR calculado para cada repetição, utilizando os dados originais das populações. Média de cinco repetições. $* * \mathrm{FR}=\mathrm{P} 12 / \mathrm{P} 6$.

pode-se afirmar ser seguro o uso conjunto desses dois importantes insumos da agroindústria, com ganhos em mão-de-obra, para as condições e produtos utilizados. $\mathrm{Na}$ Tabela 5, observa-se que as populações de $P$. zeae no solo da área irrigada e da não irrigada, aos seis meses, estavam em média maiores do que as das Pi. Aos 12 meses, os FR mostraram que as populações aumentaram de 5,31 a 15,21 vezes e nas raízes (P12/P6) de 0,61 a 3,67 o que representa altos valores, caracterizando, naquele momento, ausência de controle populacional, o qual irá induzir falta de proteção às socas. Nas raízes, o nematicida aldicarb proporcionou menores FR. Dados semelhantes foram obtidos na área não irrigada, com menores valores numéricos. Situação semelhante foi verificada em relação à população mista de Meloidogyne (Tabela 6). Tratamentos e testemunhas não diferiram em valores absolutos de Pol e PCC (Tabela 7), mostrando isenção de efeito dos tratamentos na produtividade industrial, concordando com os dados de Moura et al. (1998), Barros et al. (2000) e Rosa et al. (2003).

Como conclusões, considerando-se as condições ocorridas, tem-se que os nematicidas e herbicidas estudados foram compatíveis quando aplicados conjuntamente, no momento do plantio, não tendo sido constatadas perdas de eficácia no controle de plantas daninhas e tampouco dos nematicidas sobre as populações dos nematóides em estudo. A aplicação dos nematicidas foi efetiva apenas por proporcionar aumentos em brotação e perfilhamento em área irrigada, fatos que não se revelaram nos dados finais de produtividade. No que concerne a ação 
Estudos sobre aplicações conjuntas de herbicidas e nematicidas...

TABELA 6 - Número de Meloidogyne spp. na rizosfera e raízes da variedade de canade-açúcar (Saccharum spp.) em área irrigada e não irrigada, na presença ou ausência de dois nematicidas sistêmicos e quatro herbicidas

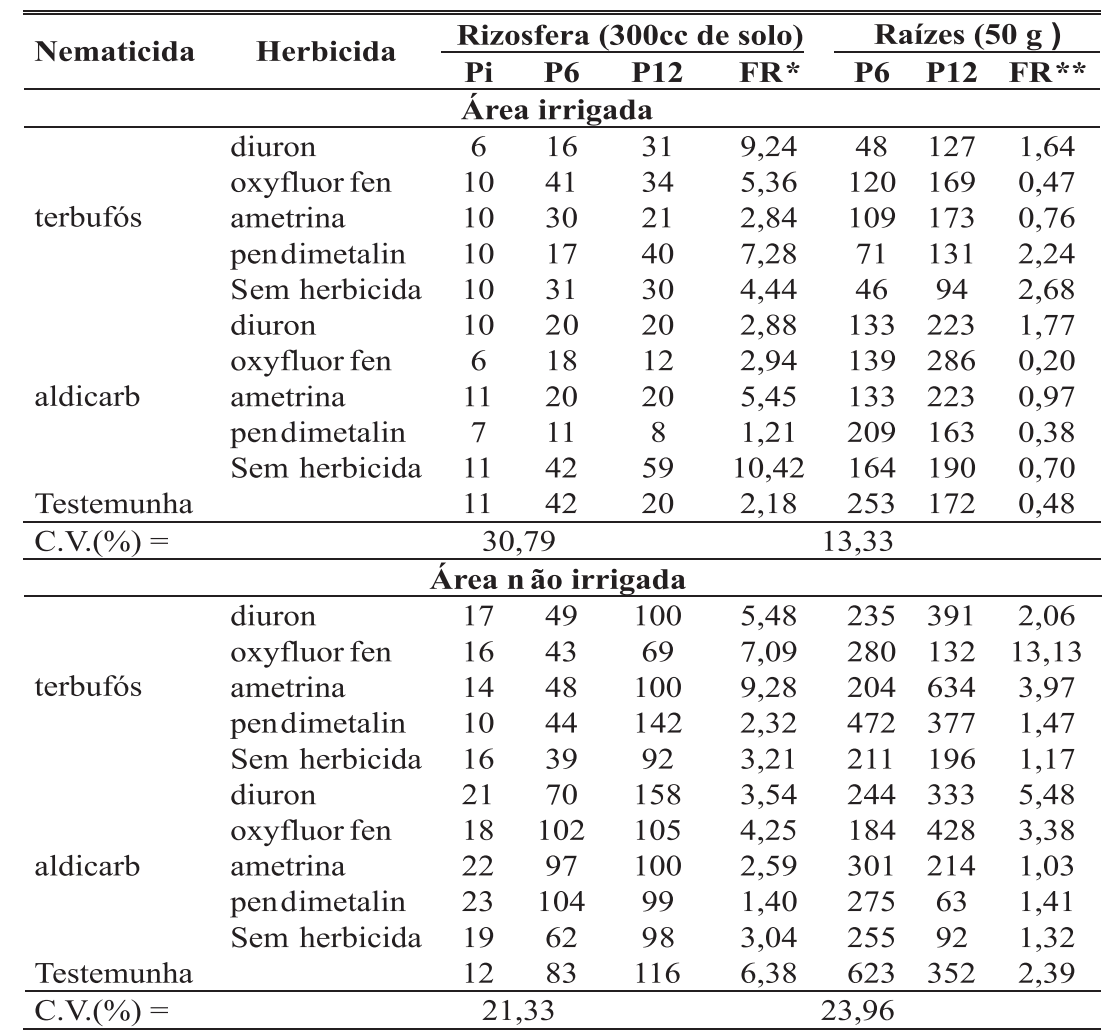

Dados apresentados em antilog $\mathrm{x}-1$. Pi = População inicial; P6 e P12 = População aos seis e 12 meses após o plantio, respectivamente. Não houve diferença significativa entre os tratamentos.

*FR $=$ P12/Pi - Média resultante das médias dos FR calculado para cada repetição, utilizando os dados originais das populações. Média de cinco repetições.

$* * \mathrm{FR}=\mathrm{P} 12 / \mathrm{P} 6$

TABELA 7 - Efeito da aplicação conjunta de nematicidas sistêmicos e herbicidas na produtividade industrial da variedade de canade-açúcar (Saccharum spp.) SP79-1011, em solos naturalmente infestados por fitonematóides, em área irrigada e não irrigada

\begin{tabular}{llcccc}
\hline \hline \multirow{2}{*}{ Nematicida } & \multirow{2}{*}{ Insumo } & \multicolumn{4}{c}{ Produtividade industrial } \\
\cline { 3 - 4 } & & Área irrigada & Área não irrigada \\
\cline { 3 - 4 } terbufós & Pol & PCC & Pol & PCC \\
\hline \multirow{4}{*}{ aluron } & 6,56 & 13,09 & 16,57 & 15,76 \\
& oxyfluorfen & 6,84 & 12,80 & 16,16 & 15,82 \\
& ametrina & 6,95 & 13,17 & 15,87 & 15,28 \\
& pendimetalin & 7,96 & 13,31 & 15,48 & 15,60 \\
& Sem herbicida & 6,36 & 12,65 & 15,64 & 15,28 \\
\hline \multirow{4}{*}{ aldicarb } & diuron & 6,80 & 13,19 & 15,51 & 12,29 \\
& oxyfluor fen & 6,44 & 13,02 & 15,85 & 15,51 \\
& ametrina & 6,89 & 13,14 & 15,05 & 15,50 \\
& pendimetalim & 7,18 & 12,78 & 15,32 & 15,17 \\
\hline Testemunha absoluta & 5,28 & 12,54 & 14,88 & 15,06 \\
\hline C.V.(\%) $=$ & Sem herbicida & 5,98 & 13,28 & 15,31 & 15,50 \\
\hline
\end{tabular}

Números médios de dez colmos tomados ao acaso. Os dados são médias de cinco repetições.

Não houve diferença significativa entre os tratamentos.

$\mathrm{Pol}=$ Percentual de sacarose .

$\mathrm{PCC}=$ Percentual de sacarose corrigida. nematicida, aferida seis meses após o plantio, não foi constatada ação de controle, ao contrário, os índices estavam e permaneceram crescentes até o momento da colheita.

\section{REFERÊNCIAS BIBLIOGRÁFICAS}

BARKER, K.R. Sampling Nematode Communities. In: Barker, K.R., Carter, C.C. \& Sasser, J.N. (Eds.) An Advanced Treatise on Meloidogyne, vol. II. Methodology. North Carolina State University Graphics. pp 3-17. 1985.

BARROS, A.C.B., MOURA, R.M. \& PEDROSA, E.M.R. Aplicação de terbufós no controle de Meloidogyne incognita raça 1 e Pratylenchus zeae em cinco variedades de cana-de-açúcar no Nordeste. Parte 1 - Efeitos na cana planta. Nematologia Brasileira 24:73-78. 2000.

BLANCO, H.G., COLETI, J.T.,MATTOS, A. O. \& OKUNO, M.S. Fitotoxidade em cana-de-açúcar causada pela interação de inseticida e herbicida residual. O Biológico 46:235-240. 1980.

COPERSUCAR. Nematóides parasitos da cana-de-açúcar e seu controle. In: Seminário de Tecnologia Agronômica, I, Piracicaba, 
SP, Anais. pp.133-153. 1982.

DINARDO-MIRANDA, L.L., NOVARETTI, W.R.T., MORELLI, J.L. \& NELLI, E.J. Comportamento de variedades de cana-deaçúcar em relação à Meloidogyne javanica em condições de campo. Nematologia Brasileira 19:60-66. 1995.

DINARDO-MIRANDA, L.L., GARCIA, V., JACON, J.J. \& COELHO, A.L. Efeitos da interação entre nematicidas e herbicidas em cana-de-açúcar. Nematologia Brasileira 25:197-203. 2001.

FERNANDEZ, A.C. Cálculo na agricultura da cana-deaçúcar. Piracicaba, SP. Edição do autor. 2001

FUGIWARA, S.M. \& CHRISTOFFOLETI, P.J. Efeito fitotóxico na cultura da cana-de-açúcar (Saccharum sp.) causado pela interação entre nematicidas e herbicidas. Resumos, Simpósio de Iniciação Científica, ESALQ, Universidade de São Paulo. 1996. p. 457.

GARCIA, V., SILVA, S.F. \& DINARDO-MIRANDA, L.L. Comportamento de variedades de cana-de-açúcar em relação a Meloidogyne incognita. Revista Nacional do Álcool e Açúcar 17:14-19. 1997.

JENKINS, W.R. A rapid centrifugal-flotation techinique for separating nematodes from soil. Plant Disease Reporter 48:692. 1964.
LUSVARGUI, H.N., PAGGIARO, C.M. \& SANTOS, J.M. Eficácia de terbufós em duas formulações e de carbofuran no manejo de nematóides e cupins em cana-de-açúcar. Resumos, 20. Congresso Brasileiro de Nematologia, Gramado, RS. 1997. p. 71.

MOURA, R.M. Dois anos de rotação de cultura em campos de cana-de-açúcar para controle da meloidoginose. 2. Considerações sobre o método e reflexos na produtividade agro-industrial da cana planta. Fitopatologia Brasileira 20:597-600. 1995.

MOURA, R.M., MACEDO, M.E.A., SILVA, E.G. \& SILVA, I.P, Efeito da aplicação de Carbofuran em cana-de-açúcar variedade CB45-3. Fitopatologia Brasileira 23:503. 1998. (Resumo)

NOVARETTI, W.R.T., NELLI, E.J. \& CARDERÁN, J.O. Testes de novos nematicidas em cana-de-açúcar. Nematologia Brasileira 9:123-133. 1985.

ROSA, R.C.T., MOURA, R.M. \& PEDROSA, E.M.R. Efeitos da Crotalaria juncea e carbofuran observados na colheita de cana planta. Nematologia Brasileira 27:167-171. 2003.

SPAULL, V.W. \& CADET, P. Nematode parasites of sugarcane. In: Luc, M., Sikora, R.A. \& Bridee, J. (Eds.) Plant Parasitic Nematodes in Subtropical and Tropical Agriculture. Wallingford. CAB International. 1990. pp. 461-491. 